\title{
Measurement of Rain Attenuation of Microwaves at 12.25GHz in Korea
}

\author{
Dong You Choi \\ Research Institute of Energy Resources Technology, Chosun University \\ dy_choi@hanmail.net
}

\begin{abstract}
We present here the results of the measurements of rain-induced attenuation in the vertically polarized signal propagating at $12.25 \mathrm{GHz}$ during some rain events, which occurred in the rainy season of the year 2001 at Yongin, Korea. The attenuation measured experimentally was compared with that obtained using the International Telecommunication Union Radio Communication Sector (ITU-R) model, the SAM model and the Global model. Our measured results are in good agreement with the ITU-R prediction.
\end{abstract}

\section{Introduction}

Rain attenuation degrades the performance of the microwave communication system and restricts the use of microwave frequencies for line of sight and space-to-earth communication link. Although fog, clouds and dust particles effect the propagation of signals significantly but rain is the major factor that adversely effects the propagation of signals at frequencies above 10GHz.[1] The knowledge of rain attenuation statistics for the frequency of operation at a particular location is very useful for the planning and engineering of a reliable satellite communication systems.[2]

Thus this paper selected Koreasat- $3\left(116^{\circ} \mathrm{E}\right)$, which uses the Ku-band $(14 \mathrm{GHz} / 12 \mathrm{GHz})$ frequency, and analyzed the beacon signal level data according to the rain rate from June to August in 2001. Then to provide analysis, the experimental data was compared with that obtained using typical rain attenuation models, such as the ITU-R model[3,4], the SAM model[5] and the Global model[6], which have previously been used in satellite communication systems.

\section{Experimental Setup and Measurement Procedure}

To measure the rain rate, a rain measurement system was used, which was installed when the Yong-in Satellite Control Office was established. The experimental site in Yong-in, Korea is at $37^{\circ} 43^{\prime} \mathrm{N}$ and about $142 \mathrm{~m}$ above sea level. To measure the beacon signal level which is always received at a certain level from a satellite, the controlling equipment of Koreasat-3 was used. Block diagrams for the two measurement systems are shown Fig. 1.

As shown in Fig. 1 (a), the accumulated rain rate data is first collected in a data collector and then is saved in a computer. The rain rate data may be saved in either 10 minute or $10 \mathrm{sec}$ intervals. Since the 10 minute interval data collection was not 
sufficiently accurate for use with the satellite beacon signal level in accuracy, the 10 sec interval data collection was used. The beacon signal, which is received at a certain level from a satellite, saves the received signal level at intervals of 1 minute, as shown in Fig. 1 (b).

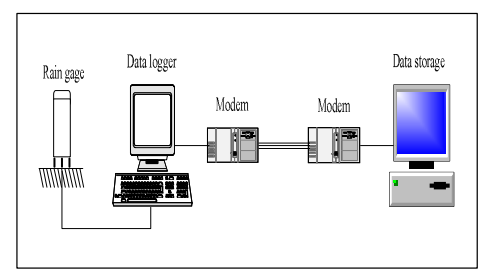

(a)

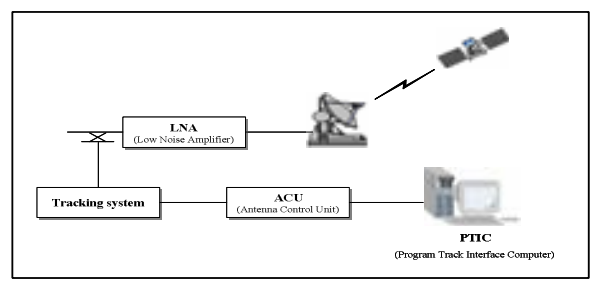

(b)

Fig. 1. Experimental system for measuring (a) the rain rate (b) the beacon signal level

The beacon signal receiving antenna used cassegrain antenna designed specifically for Koreasat-3. In order to compensate for the changes in power level caused by the perturbation of the satellite transmission, which is located in a geostationary orbit, a tracking system using the steptrack tracking method was used.

Table 1. Specifications of antenna

\begin{tabular}{||l|l||}
\hline \multicolumn{1}{|c|}{ Parameter } & \multicolumn{1}{c|}{ Characteristic } \\
\hline Diameter & $7.2 \mathrm{~m}$ \\
\hline Frequency band & $14.0 \sim 14.8 \mathrm{GHz}($ Transmit $) . \quad 11.7 \sim 12.75 \mathrm{GHz}($ Receive $)$ \\
\hline G/T at $20^{\circ} \mathrm{EL}$ & $33.5 \mathrm{~dB} / \mathrm{K} @ 11.7 \mathrm{GHz}$ \\
\hline Polarization & Dual Linear Polarized(Transmit, Receive) \\
\hline Gain & $>58.4 \mathrm{dBi} @ 14.25 \mathrm{GHz}$ \\
\hline Receive gain & $>56.2 \mathrm{dBi} @ 11.7 \mathrm{GHz}$ \\
\hline VSWR & $1.25: 1$ Maximum(Transmit, Receive $)$ \\
\hline Cross poll isolation & $>35 \mathrm{~dB}$ over $1 \mathrm{~dB}$ BW \\
\hline Sidelobes & $<-14 \mathrm{~dB}$ \\
First sidelobe & $5 \log ($ Degree $) \mathrm{dBi}$ for $1<($ Degree $)<20$ \\
Envelope & $35 \mathrm{dBi}$ for $20<($ Degree $)<26.3$ \\
$1 \sim 20^{\circ}$ & \\
\hline
\end{tabular}

Attenuation due to rain over the path was estimated by measuring the excess attenuation over clear weather attenuation values at various rain rates recorded using a tipping bucket rain gauge(diameter $200 \mathrm{~mm}$, resolution $0.5 \mathrm{~mm}$ ), which usually provides a good approximation to the instantaneous rain rates. The attenuation of radio wave propagation in a volume of rain of extent $\mathrm{L}$ in the direction of wave propagation can be expressed as [7]

$$
A=\alpha L \quad[\mathrm{~dB}]
$$

where $\alpha$ is the specific attenuation of the rain volume, expressed in decibels per kilometer. Specific attenuation can be calculated using raindrop size distribution. The relation of specific attenuation $\alpha$ and rain rate $\mathrm{R}$, is given by Olsen et al. [8] as 


$$
\alpha=a R^{b} \quad[\mathrm{~dB} / \mathrm{km}]
$$

The values of the coefficient $a$ and $b$ depend upon frequency, rain temperature and drop size distribution.

\section{Results}

\subsection{Received Beacon Signal Level and Rain Rate}

Data corresponding to the signal levels and rain rates during the propagation and communication times were collected and analyzed during the period from June to August, 2001. Rain attenuation is obtained by subtracting a reference level from the measurement of the received beacon signal level. The reference level is obtained by averaging the beacon signal level data obtained during a period in which there is no rain. The 1 st to 8 th set of statistical data represent the mean values of the data collected on rainy days at intervals of 3 4 days after excluding the minimum and maximum values. The total statistical data was obtained by summing all of the mean data values during each measurement period. Fig. 2 shows the rain attenuation according to rain rate, which was both, measured and calculated using the rain attenuation prediction model.

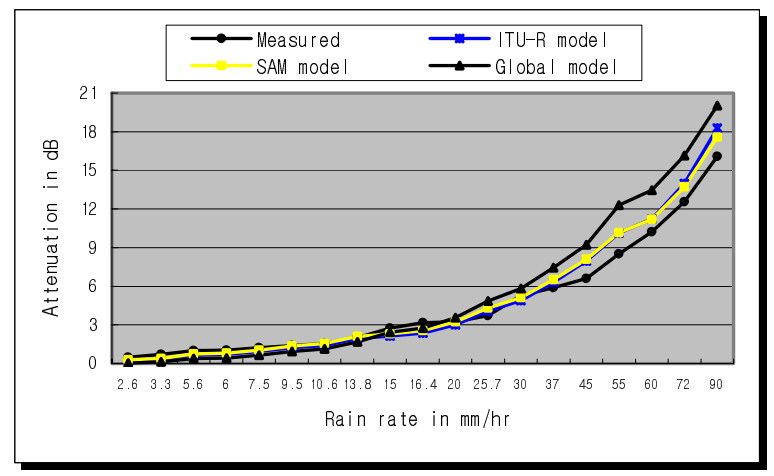

Fig. 2. Comparison of calculated attenuation and measured attenuation

\subsection{Discussion}

It can be seen in Fig. 2 above, that the experimentally measured attenuation at $12.25 \mathrm{GHz}$ is in agreement with that calculated using the ITU-R model, the SAM model, and the Global model for the measured rain rates.

The results shown in Fig. 2 show that, the ITU-R model was in good agreement with the measured attenuation up to a rain rate of about $20 \mathrm{~mm} / \mathrm{hr}$. When the rain rate exceeds $20 \mathrm{~mm} / \mathrm{hr}$, the ITU-R model was in excellent agreement with the measured attenuation. As shown in Fig. 2, the SAM model was in excellent agreement with the 
measured attenuation up to about $20 \mathrm{~mm} / \mathrm{hr}$, however above this value there is a difference in the attenuation value of above $0.17 \sim 1.5 \mathrm{~dB}$ up to the measured rain rate of $60 \mathrm{~mm} / \mathrm{hr}$. The Global model shown in Fig. 2 underestimates the attenuation up to a rain rate of about $20 \mathrm{~mm} / \mathrm{hr}$ and, above that rate, it overestimates the attenuation as compared with our experimental results.

\section{Conclusions}

The rain-induced attenuation at $12.25 \mathrm{GHz}$ was measured experimentally. It was found that the experimental results were in good agreement with those obtained using the ITU-R model. At low rain rate, the experimental results were also in close agreement with the SAM model. However, the Global model either underestimates or overestimates the experimentally measured attenuation at the same rain rate. From the above observations, it can be concluded that the rain-induced attenuation at $12.25 \mathrm{GHz}$ can be satisfactorily predicted using the ITU-R model for this latitude. However, the raininduced attenuation statistics cannot be predicted satisfactorily using the SAM model or the Global model for the present location.

\section{References}

1. T. C. Ramadorai: Rain attenuation and prediction in the Satellite-Earth Path. in Proc. Workshop HF VHF and Microwave Communications, New Delhi, India(Feb. 1987)

2. Ashok Kumar and I. S. Hudiara: Measurement of Rain-Induced Attenuations of Microwaves at 19.4GHz. IEEE Transactions on Communications, Vol. 1, (JAN., 2002.) 84-86

3. ITU-R: Propagation data and prediction methods required for the design of Earth-space Telecommunications systems. Rec. P.618, ITU-R(1997)

4. ITU-R: Specific attenuation model for rain for use in prediction methods. Rec. P.838, ITU$\mathrm{R}(1999)$

5. W. L. Stutzman and W. K. Dishman: A simple model for the estimation of rain-induced attenuation along earth-space paths at millimeter wavelength. Radio Science, Vol. 17, no. 6(Nov.-Dec., 1982) 1465-1476

6. R. K. Crane: Prediction of attenuation by rain. IEEE Transactions on Communications, Vol. COM-28, no.9(Sept, 1980) 1717-1733

7. CCIR: Propagation data and prediction methods required for earth-space telecommunication systems. in proc. Plenary Assembly, Vol. 5, Geneva, Switzerland, Rep. 564-4(1990) $447-$ 505

8. R. L. Olsen, D. V. Rogers, and D. B. Hodge: The $a R^{b}$ relation in the calculation of rain attenuation. IEEE Trans. Antennas propagation, Vol. AP-26(1978) 318-329 